\title{
Diagnostic et évaluation des états de conscience altérée
}

\author{
Caroline Schnakers ${ }^{(1,2)}$, Steve Majerus ${ }^{(2)}$, Steven Laureys ${ }^{(1,2)}$ \\ ${ }^{1}$ Centre de Recherches du Cyclotron; \\ ${ }^{2}$ Département de Sciences Cognitives; Université de Liège, Belgique
}

\section{Résumé}

Ces dernières années, le nombre de patients en état de conscience altérée a fortement augmenté. En effet, avec le perfectionnement des techniques de réanimation, de nombreux patients sévèrement cérébrolésés survivent à leurs lésions. Néanmoins, tandis que certains se rétablissent rapidement, d'autres prennent plus de temps et traversent différents états de conscience altérée (tels que le coma, l'état végétatif, ou l'état de conscience minimale) avant de récupérer ou non un état de conscience normal. Il n'existe cependant aucun moyen direct d'évaluer la conscience. L'évaluation comportementale constitue le principal moyen de mesure. De nombreuses échelles standardisées (par exemple, la Glasgow Coma Scale, la Wessex Head Injury Matrix ou la Coma Recovery Scale) ont été développées dans cette optique. Elles donnent des informations précieuses quant à la progression de l'état de conscience de ces patients et permettent d'adapter la prise en charge de ceux-ci. D'autres techniques peuvent être utilisées de manière complémentaire. La tomographie à émission de positons (PET scan) peut aider lors du diagnostic en apportant des données utiles sur le fonctionnement cérébral des patients en état de conscience altérée.

Mots-clés: état de conscience altérée - diagnostic - évaluation comportementale - imagerie fonctionnelle

\begin{abstract}
Progress in intensive care has increased the number of patients who survive severe acute brain injury. Nevertheless, whereas some patients recover quickly, others take more time and pass through different states of altered consciousness (coma, vegetative state, minimally conscious state) before recovering a normal state of conscious awareness. Still other patients will remain in a more permanent state of altered consciousness. The management of these patients is thus very important. However, no methods can measure awareness directly. The bedside behavioural assessment is the main way to detect signs of awareness. In his context, a large number of scales have been developed (for instance, the Glasgow Coma Scale, the Wessex Head Injury Matrix or the Coma Recovery Scale). These give useful information about the patient's recovery and permit to adapt the patents' daily management. Additionally, functional neuroimaging techniques (e.g., positron emission tomography PET or functional magnetic resonance imaging - fMRI) can be used to objectively measure brain function in these patients. Combining extensive neuropsychological testing with functional neuroimaging techniques permits to better estimate the residual cognitive capacities and their evolution over time.
\end{abstract}

Key words: altered states of consciousness - behavioural assessment - brain imaging

\section{Introduction}

De nombreux patients sont admis aux soins intensifs avec une atteinte cérébrale ou subissent des complications au niveau neurologique qui les plongent dans un état de conscience altérée. L'évaluation de l'état de conscience dés l'entrée du patient et tout au long de son hospitalisation est essentielle pour une prise en charge adaptée. Ces évaluations permettent d'obtenir des informations précieuses quant à la progression du patient. Il n'existe néanmoins aucun outil pouvant évaluer directement la conscience. L'observation comportementale constitue le principal moyen de détecter les signes de conscience. Une distinction doit tout d'abord être faite entre 1' 'état de vigilance' (présence d'un cycle veille-sommeil caractérisé par l'ouverture spontanée de yeux) et 1'état de conscience' (par exemple, la réponse à un ordre verbal). En effet, un patient peut être vigilant mais ne montrer aucun signe évident de conscience. Le patient en état végétatif en est un bon exemple: ses yeux s'ouvrent de manière spontanée, son cycle veille-sommeil est préservé pourtant il n'obéit à aucun ordre verbal, aucun signe de 
conscience n'est observé. L'éveil est une condition nécessaire mais insuffisante au phénomène de conscience. La conscience n'est, d'autre part, pas un phénomène unique. On différencie souvent la conscience de soi (c'est-à-dire la conscience de ses propres pensées) de la conscience de son environnement (c'est-à-dire la perception consciente du monde environnant). Néanmoins, la conscience de soi reste difficile à percevoir. Seule la personne elle-même sait qu'elle est consciente. L'évaluation au chevet du patient en état de conscience altérée se limite donc essentiellement à une observation de la conscience de l'environnement (par exemple, en observant s'il y a réponse à un ordre verbal simple tel que 'Serrez ma main').

\section{Les états de conscience altérée}

Lors de ces évaluations, différents états de conscience altérée tels que la mort cérébrale, le coma, l'état végétatif et l'état de conscience minimale sont à distinguer (tableau 1 et figure 1).

La mort cérébrale se caractérise par une perte de toutes les fonctions du tronc cérébral. Elle s'apparente à un coma irréversible où le patient est apnéique et totalement a-réactif à son entourage [18]. Un examen électroencéphalographique permet d'objectiver un tracé isoélectrique reflétant l'arrêt de l'activité électrique cérébrale. D'autres techniques tel que le doppler transcrânien montrent l'arrêt de perfusion sanguine au niveau du cerveau. Après avoir exclu une dépression cérébrale par drogues ou hyperthermie, un diagnostic définitif peut être établi après une période de 6 à 24 heures.

\section{ÉTAT NORMAL \\ COMA}

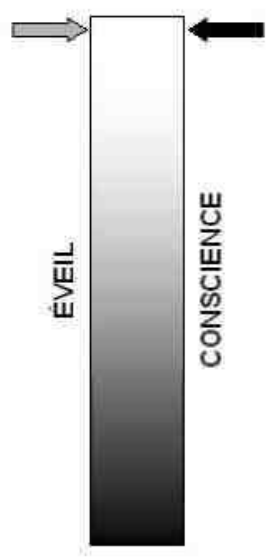

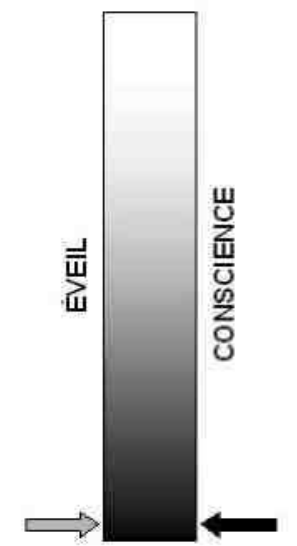

ÉTAT VÉGÉTATIF

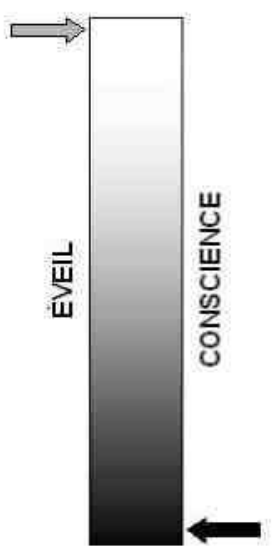

\section{ÉTAT DE CONSCIENCE} MINIMAL

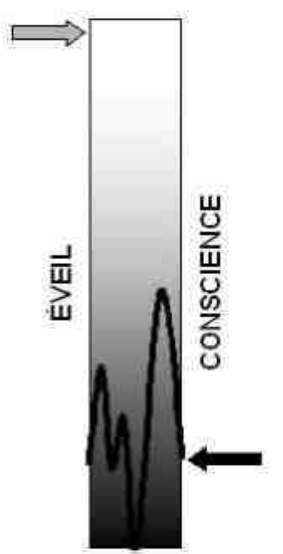

\section{SYNDROME LOCKED-IN}

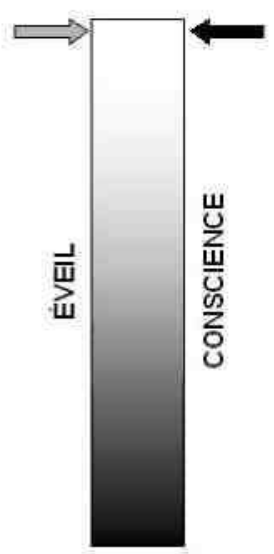

Fig. 1 : La conscience a deux composantes : l'éveil et la conscience de soi et du monde extérieur. Dans le coma, aucune de ces deux composantes n'est présente. Dans l'état végétatif, la personne est éveillée, mais n'a aucune conscience de soi ni de son environnement. Une personne dans un état de conscience minimale est éveillée et présente parfois des signes fugaces d'actions conscientes. Enfin, une personne ayant un syndrome locked-in est éveillée, parfaitement consciente, mais, comme elle est paralysée, elle communique uniquement par des clignements des yeux avec son entourage.

Le coma résulte d'une dysfonction au niveau du cortex cérébral bilatéral ou du système réticulé activateur du tronc cérébral [19]. Le patient comateux n'ouvre pas les yeux (ni spontanément ni après une stimulation) et, même après ouverture manuelle, aucune poursuite visuelle n'est observée [1]. Il n'émet aucun son et n'obéit à aucun ordre verbal. Aucune réponse volontaire et consciente n'est observée. Seule, une activité réflexe persiste. Cet état doit durer au moins une heure pour être différencié d'une syncope ou d'un autre état de conscience transitoire. Une tomodensitométrie (CT scan) permettra ensuite de définir l'étendue des lésions cérébrales. Cependant, l'imagerie par résonance magnétique (IRM) sera plus informative si les lésions sont de petites tailles. Le tracé électroencéphalographique est souvent ralenti de manière globale ; il constitue un outil utile pour suivre l'évolution des patients comateux. Les comas prolongés sont rares. Le coma peut durer de 2 à 4 semaines puis évoluer vers un état végétatif ou, rarement, un syndrome locked-in. Le pronostic est généralement posé endéans 3 jours, pour les comas d'origne non-traumatique. La moitié des patients sans chance de récupération décède durant cette période. La majorité des patients restant (90 à 95\%) récupère endéans 3 jours à 2 semaines tandis que 50 à $80 \%$ des traumatisés crâniens récupèrent après un an [2]. 
Tableau 1. Caractéristiques de différents états de conscience altérée

\begin{tabular}{|c|c|c|c|}
\hline Etat & Critères diagnostique & EEG & FDG-PET \\
\hline Mort cérébrale & $\begin{array}{l}\text { absence d'éveil } \\
\text { absence de conscience } \\
\text { absence de fonctions respiratoires } \\
\text { perte des réflexes du tronc cérébral }\end{array}$ & isoélectrique & aucune activité \\
\hline Coma & $\begin{array}{l}\text { absence d'éveil } \\
\text { absence de conscience } \\
\text { fonction respiratoire variable } \\
\text { présence variable des réflexes du } \\
\text { tronc cérébral } \\
\text { aucune production de sons }\end{array}$ & $\begin{array}{l}\text { ralentissement } \\
\text { généralisé important }\end{array}$ & 40 à $50 \%$ diminution \\
\hline Etat végétatif & $\begin{array}{l}\text { éveil (ouverture spontanée des } \\
\text { yeux) absence de conscience } \\
\text { souvent fonction respiratoire } \\
\text { préservée préservation des réflexes } \\
\text { du tronc cérébral parfois } \\
\text { verbalisations non significatives }\end{array}$ & $\begin{array}{l}\text { ralentissement } \\
\text { généralisé important }\end{array}$ & $\begin{array}{l}50 \text { à } 60 \% \\
\text { diminution } \\
\text { (zones associatives) }\end{array}$ \\
\hline $\begin{array}{l}\text { Etat de } \\
\text { conscience } \\
\text { minimale }\end{array}$ & $\begin{array}{l}\text { éveil (ouverture spontanée des } \\
\text { yeux) conscience minimale } \\
\text { (réponse inconsistante à un ordre } \\
\text { verbal) fonction respiratoire } \\
\text { préservée préservation des réflexes } \\
\text { du tronc cérébral verbalisations } \\
\text { possibles mais élémentaires }\end{array}$ & $\begin{array}{l}\text { ralentissement } \\
\text { généralisé }\end{array}$ & 20 à $40 \%$ diminution \\
\hline $\begin{array}{l}\text { Locked-in } \\
\text { syndrome }\end{array}$ & $\begin{array}{l}\text { éveil (ouverture spontanée des } \\
\text { yeux) } \\
\text { conscient } \\
\text { (communication par mouvements } \\
\text { des yeux) } \\
\text { souvent fonction respiratoire } \\
\text { préservée } \\
\text { préservation des réflexes du tronc } \\
\text { cérébral } \\
\text { verbalisations impossibles } \\
\text { (anarthrie) } \\
\text { tétraplégie }\end{array}$ & normal & activité normale \\
\hline
\end{tabular}

L'état végétatif résulte souvent d'une atteinte globale du cortex ou de la matière blanche ou, dans certains cas, d'une lésion thalamique bilatérale avec préservation de la substance réticulée [23]. Le terme 'végétatif' signifie qu'il y a préservation des fonctions autonomes (régulation cardio-vasculaire, thermorégulation) et du cycle veille-sommeil (épisodes d'ouverture spontanée des yeux). Le patient ne répond pas à la commande verbale et, s'il peut émettre des grognements, il ne parle pas et ne produit aucun mot. Dans certains cas, des comportements tels que rire, pleurer ou grimacer sans raison apparente ou suivre un objet des yeux pendant une très courte durée (1-2 secondes) ont été observés. La compatibilité du suivi du regard avec le diagnostic d'état végétatif est encore débattue. Néanmoins, aucun comportement de ce type de patient n'est volontaire et dirigé dans un but précis. Ils ne reflètent aucun signe de conscience mais plutôt une activité réflexe [25]. Etablir un pronostic est difficile. Un jeune âge, une étiologie traumatique et peu de temps passé en état végétatif améliorent le pronostic [23]. Lorsque le patient reste un mois ou plus dans cet état, on parle d'état végétatif "persistant" [10] et "permanent" après une période de 3 mois (pour une étiologie non-traumatique) à un an (pour une étiologie traumatique) [23].

Cependant, cette dernière appellation sous-entend une quasi-absence de récupération et peut donner lieu à de graves décisions (comme le retrait de tout traitement médical ou de toute alimentation) alors qu'il est impossible de certifier l'absence de récupération. Il est donc préférable de préciser, seulement à titre indicatif, la durée en mois [1] et d'omettre les étiquettes «persistant» ou « permanent». 
L'état de conscience minimale se caractérise, contrairement à l'état végétatif, par un certain degré de conscience. Un patient en état de conscience minimale présente des réponses reproductibles à la commande et peut suivre des yeux un objet de manière soutenue [7]. Le patient peut aussi communiquer de manière élémentaire mais adéquate, et ceci par le canal verbal ou gestuel (par un mouvement de la tête signifiant « oui » ou « non »). Il peut manifester des comportements émotionnels adaptés, contrairement aux patients en état végétatif qui pleurent et rient de manière non pertinente [1]. Il est important de souligner que ces réponses sont souvent fluctuantes. Elles peuvent être observées puis absentes d'un jour à l'autre ou selon le moment de la journée (matin ou après-midi). La réponse à une stimulation ou à un ordre verbal est d'autre part souvent latente. Une sollicitation importante et répétée est parfois indispensable. Cette réponse devra, en tout cas, être reproductible (c'est-à-dire observée plusieurs fois) avant de conclure à une action volontaire. Une récupération de l'état de conscience va de pair avec des réponses de plus en plus consistantes. Le patient est sorti de l'état de conscience minimale lorsqu'il est capable de communiquer de manière fonctionnelle [7]. Comme pour l'état végétatif, il est difficile d'émettre un pronostic. Les chances de récupération sont meilleures qu'en état végétatif. Cependant, certains patients ne progressent pas ou peu et restent de manière prolongée dans cet état.

Le syndrome « locked-in » (syndrome de 'dé-efférentation' ou de 'verrouillage') se caractérise par une tétraplégie, une amimie et une paralysie de l'appareil bucco-facial dues à une lésion ventro-pontine au niveau du tronc cérébral. Cet état est, la plupart du temps, la conséquence d'une thrombose basilaire mais découle parfois d'un traumatisme crânien. Ces patients sont quasi totalement paralysés mais leurs sensations sont intactes. En fait, de façon superficielle, cet état ressemble à l'état végétatif alors que les patients « locked-in » sont entièrement conscients de leur environnement et d'eux-mêmes. Classiquement, ils ont les yeux ouverts et sont quasi immobiles. Leur paralysie les empêche de répondre à des ordres moteurs. Le seul moyen qu'ils ont pour communiquer avec l'extérieur sont les mouvements des yeux. En effet, les mouvements verticaux des yeux et les clignements sont préservés [6]. Le patient en syndrome locked-in peut répondre à une série de questions par un code élémentaire (p.e., lever les yeux pour 'oui' et baisser les yeux pour 'non'. Ce code peut être établi dés la phase aiguë. D'autres moyens de communicaton plus élaborés peuvent ensuie être employés tels qu'un alphabet adapté à leur mode de communicaton (par exemple, l'alphabet ESARINT ; tableau 2) ou un appareil informatisé qui leur permet de communiquer grâce à une caméra détectant les mouvements oculaires, lesquels peuvent alors être traduits dans un code permettant de commander un ordinateur.

Tableau 2. Alphabet ESARINT : mode de communication adapté aux patients en syndrome « locked-in »
1. $\mathrm{E}$ S A $\mathrm{A}$ I $\mathrm{N}$ T

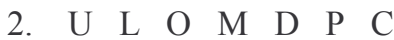

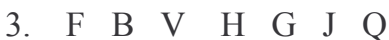
4. $\mathrm{Z} \quad \mathrm{Y} \quad \mathrm{X} \quad \mathrm{K} \quad \mathrm{W}$

Consignes: Le patient doit d'abord sélectionner la ligne dans laquelle il y a la lettre qu'il veut utiliser. A cette fin, on prononce à voix haute et lentement (un numéro par seconde) le numéro de ces lignes. Le patient clignera, par exemple, deux fois des yeux lorsque le bon numéro aura été prononcé. Ensuite, pour sélectionner la lettre, prononcer à voix haute et lentement (une lettre par seconde) chaque lettre de la ligne sélectionnée. Le patient clignera, par exemple, deux fois des yeux lorsque la lettre qu'il veut utiliser aura été prononcée. Recommencer la procédure jusqu'à former un mot ou une phrase. Remarque: l'alphabet doit être mis face au patient. Il est important de s'assurer que le patient le voit bien en lui posant la question.

Cependant, il arrive que ces patients puissent bouger autre chose que leurs yeux. En effet, la paralysie est rarement complète et certains patients récupèrent la mobilité d'une (ou plusieurs) partie(s) du corps (comme la tête ou un doigt). Cette récupération même si minime est précieuse et certaines technologies peuvent être adaptées en fonction des mouvements préservés (par exemple, une chaise roulante téléguidée par le doigt mobile) augmentant ainsi l'autonomie du patient. En fait, avec l'évolution actuelle des technologies de pointes, environ la moitié de ces patients peuvent retourner à domicile avec des soins adaptés. En outre, ils peuvent vivre longtemps (plus de 12 ans; [11]). Le suivi et la prise en charge de ces patients sont donc importants car ils sont pleinement conscients tout en étant incapables de communiquer leurs souffrances physiques ou psychologiques autrement que par mouvements oculaires. Un code élémentaire de communication (oui-non) doit donc être instauré très tôt. 


\section{Evaluation clinique de la conscience et échelles comportementales}

Même s'il existe différents états de conscience altérée, un réel clivage est difficile à faire entre ces états. Un patient dans le coma peut passer rapidement en état végétatif puis en état de conscience minimale pour retomber ensuite dans le coma. La conscience n'est pas un phénomène de tout ou rien mais elle se situe plutôt sur un continuum. Ce qui distingue ces états, c'est avant tout, leur niveau de vigilance et de conscience. Un patient peut passer par un état de coma (fermeture des yeux), de stupeur (ouverture des yeux après une importante sollicitation), être somnolent (ouverture des yeux après sollicitation) puis éveillé (ouverture des yeux spontanée). Comme nous l'avons vu plus haut, le fait d'être éveillé n'implique pas automatiquement que le patient soit conscient. Néanmoins, la barrière est ténue entre ce qui est considéré comme conscient ou non. Le clinicien doit toujours être attentif aux moindres comportements reflétant un signe de conscience. Ces comportements doivent être volontaires, reproductibles et clairement observés (ce qui, en pratique, est rarement le cas). Cest pourquoi il est important d'observer ces comportements à plusieurs reprises. Seule une évaluation systématique permet de voir l'évolution du patient de jour en jour.

De nombreux outils standardisés ont été développés pour évaluer l'état de conscience. L'outil le plus connu et le plus utilisé est sans aucun doute la Glasgow Coma Scale (GCS) [22]. Cependant, cette échelle n'est pas la seule et il en existe d'autres plus complètes et plus sensibles. Certaines sont une aide au diagnostic dans les premières heures, d'autres sont plus sensibles aux changements au cours du emps, tout au long de la récupération du patient. Le choix d'une ou plusieurs de ces échelles dépend des objectifs de l'évaluation (évaluation rapide ou détection de changements minimaux et subtils) mais aussi de l'état de conscience du patient (certaines échelles sont plus sensibles pour le coma et d'autres le sont pour l'état végétatif ou l'état de conscience minimale). Une seule échelle ne peut donc être adaptée dans toutes les situations et il convient de les choisir de manière adéquate.

La Glasgow Coma Scale (GCS) [22] est assez courte, rapidement administrable et s'intègre facilement aux soins infirmiers. Elle permet d'évaluer le patient dés son entrée à l'hôpital et de surveiller son évolution durant son hospitalisation. Elle comprend une partie motrice, verbale et visuelle. Seule la meilleure réponse observée (spontanée ou en réponse à une stimulation) à chaque sous-échelle est retenue pour la cotation et la somme de ces différentes sous-échelles constitue le score total sur 15 points. Il est cependant préférable d'utiliser le score individuel de chaque sous-échelle. La sommation masque souvent l'hétérogénéité observée au niveau des différentes sous-échelles et un seul score peut refléter des comportements et des états de conscience fort différents. En cas d'intubation ou de trachéotomie, la partie verbale ne peut pas être admninistrée et le score total ne peut plus être utilisé. Au niveau de la sous-échelle visuelle, il faut préciser s'il y a une lésion empêchant l'ouverture des yeux (par exemple, suite à un oedème périorbital, à un traumatisme de la face etc.). Ces indications permettent de ne pas biaiser l'évaluation. La GCS est particulièrement intéressante pour suivre l'évolution de patients dans le coma. Néanmoins, l'évaluation des réflexes du tronc cérébral est complémentaire. Il existe une extension de la GCS appelée la Glasgow Liège Scale [4](GLS) comprenant, en plus des 3 souséchelles de la GCS, une partie sur 5 points pour l'évaluation des réflexes du tronc cérébral (tels que frontoorbiculaire, oculo-céphalique vertical et horizontal, photomoteur et oculo-cardiaque). Cette partie fournit des informations précieuses quant à l'évolution du patient. En effet, la préservation des réflexes du tronc cérébral chez un patient comateux est de meilleur pronostic tandis qu'une dégradation peut signifier une détérioration rostro-caudale.

La Coma Recovery Scale [8](CRS; tableau 3) s'adresse à des patients en état végétatif ou en état de conscience minimale. Elle consiste en 35 items et évalue les fonctions auditives, visuelles, motrices, oromotrices/verbales, la communication et l'éveil. Les items de chacune de ces parties sont disposés hiérarchiquement du niveau le plus bas représentant des réponses réflexes au niveau le plus haut des activités corticales. De plus, tandis que la GLS s'intéresse à des réponses hiérarchiquement plus « inférieures », cette échelle évalue des fonctions plus complexes telles que la communication ou encore la prise de décision. Elle permet ainsi de mieux détecter des changements au niveau de la récupération du patient et de mieux prédire la récupération fonctionnelle. Il est cependant préférable, tout comme pour la GCS, de ne pas prendre le score total mais de se baser plutôt sur le score obtenu aux différentes sous-échelles. 
Tableau 3. Version Française de la CRS-R (Coma Recovery Scale [8] revue en 2004 ; Giacino et Kalmar, sous presse)

\section{ÉCHELLE CRS-R DE \\ RÉTABLISSEMENT DES PATIENTS \\ COMATEUX}

\section{FONCTION AUDITIVE}

4 - Mouvements cohérents sur demande*

3 -Mouvements reproductibles sur

demande*

2 - Localisation des sons

1 - Tressaillement auditif

0 - Néant

\section{FONCTION VISUELLE}

5 - Reconnaissance des objets*

4 -Localisation des objets : atteinte*

3 - Mouvements oculaires de recherche*

2 - Fixation*

1 - Tressaillement visuel

0 - Néant

\section{FONCTION MOTRICE}

6 - Utilisation fonctionnelle des objets ${ }^{+}$

5 - Réaction motrice automatique*

4 - Manipulation d'objets*

3 -Localisation des stimulations nocives*

2 - Mouvements réflexes de retrait

1 - Posture anormale

0 - Néant/Paralysie flasque

\section{FONCTION OROMOTRICE/VERBALE}

3 - Verbalisation intelligible*

2 - Vocalisation/Mouvements oraux

1 - Mouvements réflexes oraux

0 - Néant

\section{COMMUNICATION}

2 - Fonctionnelle : précise

1 - Non fonctionnelle : intentionnelle*

0 - Néant

ÉVEIL

3 - Attention

2 - Ouverture des yeux sans stimulation

1 - Ouverture des yeux avec stimulation

0 - Aucun éveil

Indique la sortie progressive de l'état de conscience minimale ${ }^{+}$ Indique un état de conscience minimale*

L'approche de la Wessex Head Injury Matrix [21] (WHIM) est aussi intéressante à considérer. Cete échelle, récemment adaptée en français [17], a été conçue pour évaluer de manière sensible l'évolution du patient et ceci de la sortie du coma jusqu'à une récupération relativement complète de la conscience et des fonctions cognitives. Elle évalue principalement l'éveil et la concentration, la conscience visuelle (c'est-à-dire la poursuite visuelle), la communication, la cognition (mémoire, orientation spatio-temporelle) et les comportements sociaux. Cependant, 
ces composantes ne sont pas divisées en sous-échelles. La WHIM est une échelle principale qui se compose de 62 items ordonnés selon une séquence hiérarchisée et reflétant la séquence moyenne de récupération observée auprès d'une grande population de patients sévèrement cérébrolélées. Le score représente le rang du comportement le plus évolué observé. On peut aussi additionner le nombre de comportements observés d'une évaluation à l'autre pour permettre une plus grande précision. Cet outil est particulièrement indiqué chez les patients en état de conscience minimal et qui ne semblent plus évoluer au niveau d'autres échelles moins sensibles (comme la GCS, par exemple). Il est vrai que l'administration de cette échelle prend plus de temps que celle d'autres échelles (de quelques minutes à plus de 30 minutes selon l'état de conscience du patient).

Cependant, la WHIM permet d'observer les déficits mais aussi les capacités préservées du patient et peut être le point de départ d'une véritable prise en charge du malade.

\section{La tomographie à émission de positons}

L'ensemble des échelles comportementales est indispensable pour un bon suivi et une intervention adéquate auprès du patient en état de conscience altérée. La tomographie à émission de positons (ou PETscan) est une technique d'imagerie qui permet de fournir des informations quant au fonctionnement cérébral des patients en état de conscience altérée. Ces dernières années, plusieurs travaux de recherche ont permis de montrer son intérêt dans le domaine.

Ainsi, pour le coma, une dépression globale du métabolisme cérébrale de 40 à $50 \%$ chez des patients comateux d'origine traumatique ou hypoxique a été montrée [24] (figure 2). La récupération n'est cependant pas toujours accompagnée d'un rétablissement complet de l'activité cérébrale et une diminution de $25 \%$ a pu être observée chez des patients ayant récupéré d'un coma post-anoxique [5]. Néanmoins, aucune corrélation n'a encore été trouvée entre la diminution du métabolisme et les chances de récupération [9].

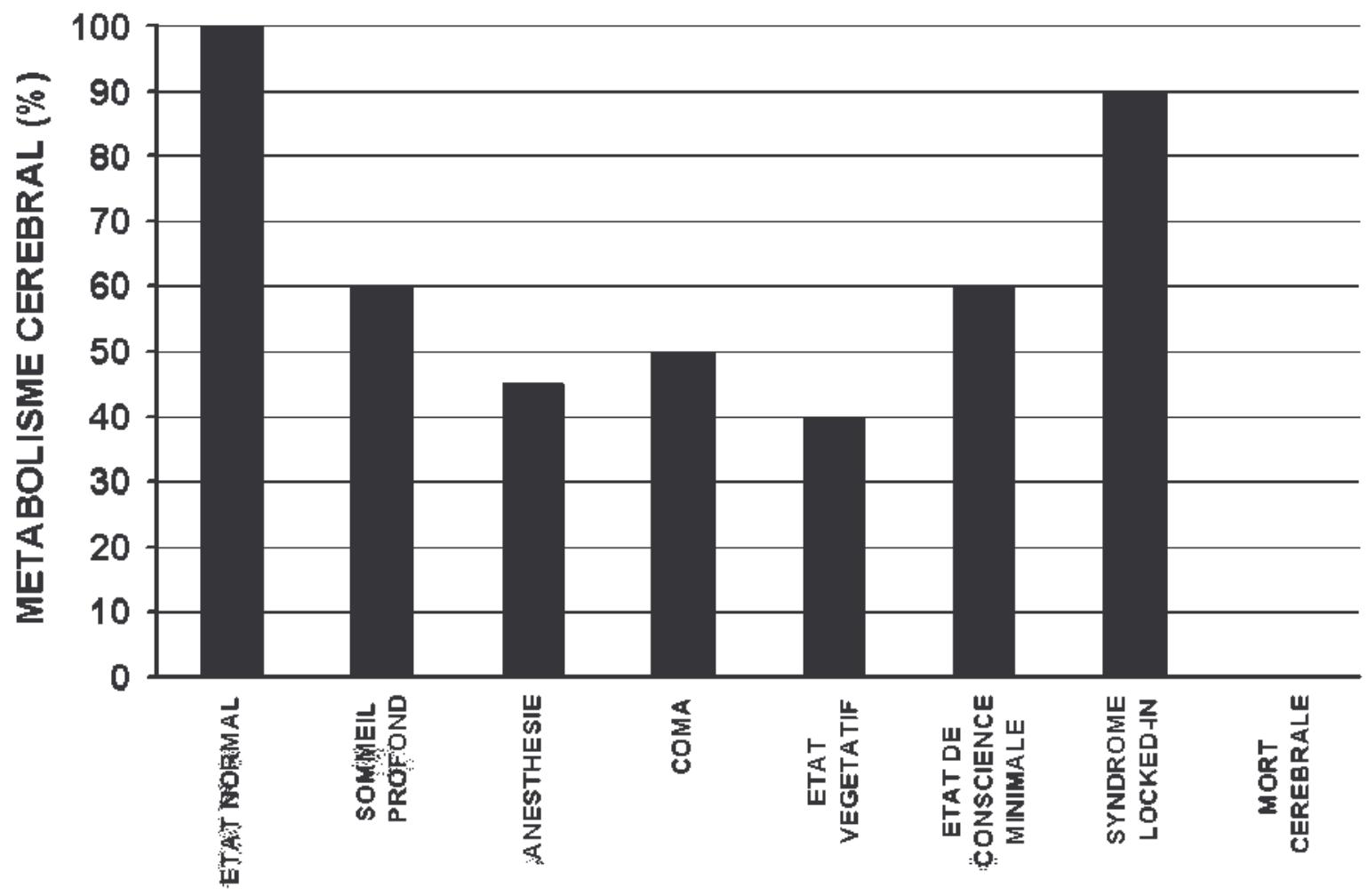

Fig. 2 : Métabolisme cérébral (consommation de gucose) dans les différentes groupes diagnostiques

Chez des patients en état végétatif, la diminution du métabolisme est de 50 à $60 \%$ et semble encore s'aggraver (60 à 70\%) pour les patients en état végétatif 'permanent' [20,24], Le temps passé semble donc préjudiciable. D'autre part, on observe une atteinte systématique des cortex associatifs connus pour être impliqués dans des processus liés à la conscience tels que l'attention, la mémoire et le langage [16] (figure 3). Il est néanmoins difficile de savoir si cette diminution vient d'une perte irréversible de neurones ou si elle est fonctionnelle et potentiellement réversible. Chez des patients ayant récupéré, on observe non seulement un retour à la normal du métabolisme dans ces régions [16] mais surtout un rétablissement de l'interaction de celles-ci avec le thalamus 
[14]. En fait, les régions activées à la suite d'une stimulation, par exemple, auditive [13] ou douloureuse [15] n'impliquent que les régions primaires et non les régions associatives indispensables à une interprétation plus élaborée du stimulus. Cette activité résiduelle suggère une absence d'intégration de l'information nécessaire à une récupération de la perception consciente.

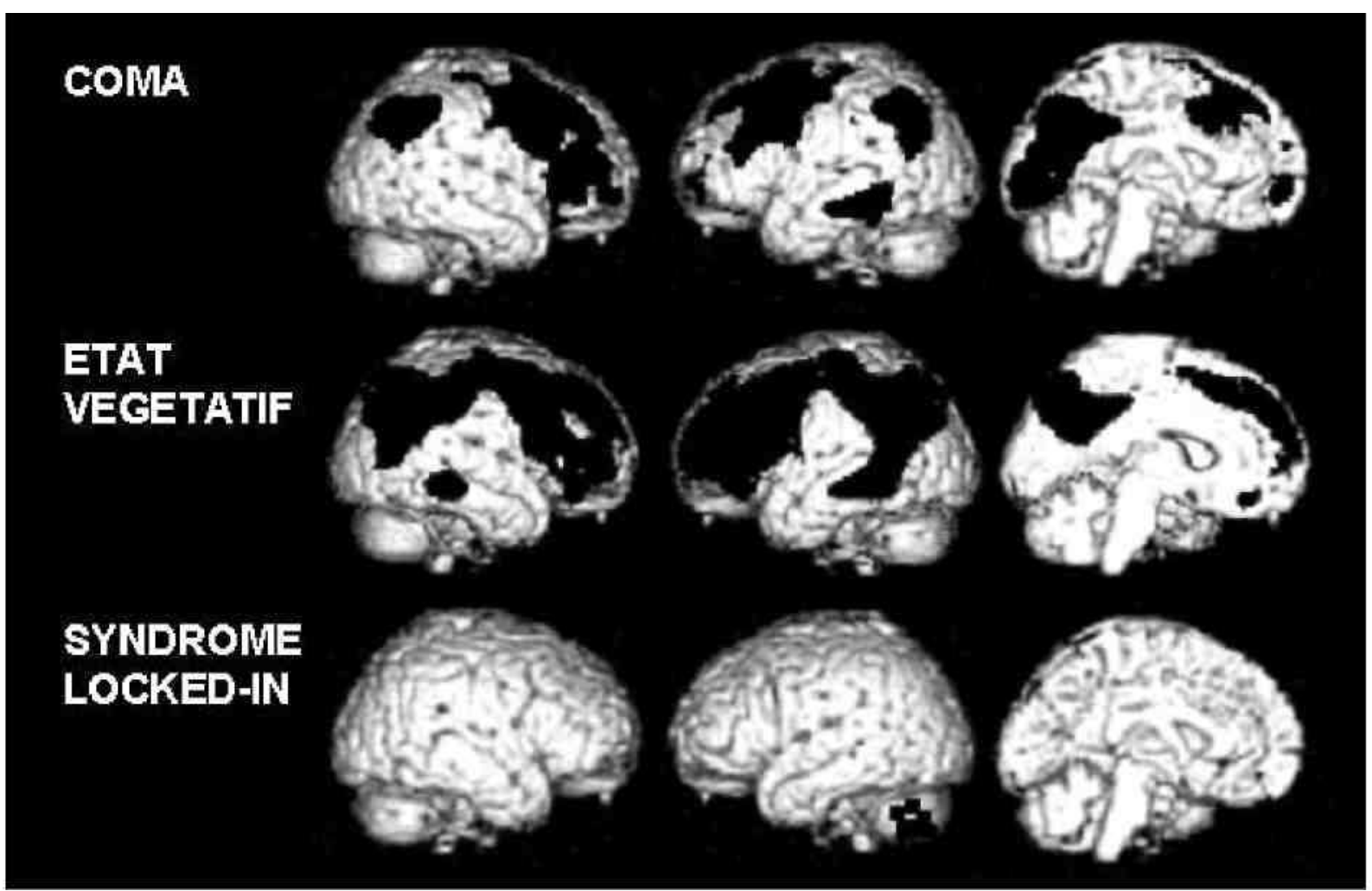

Fig. 3 : Aires cérébrales hypométaboliques (les zones en noir) dans un état de coma, dans un état végétatif et dans un syndrome locked-in. Dans le coma et dans l'état végétatif, de nombreuses aires du cortex associatif présentent un métabolisme anormal. Dans ces deux états, ce sont quasiment les mêmes aires qui sont touchées : les patients ne sont pas conscients. Au contraire, dans le syndrome locked-in, seule une aire limitée du cervelet présente un métabolisme réduit: les personnes atteintes de ce syndrome sont parfaitement conscientes, prisonnières dans un corps immobile.

Le pattern métabolique semble être différent pour le patient en état de conscience minimale. Malgré une dépression métabolique gobale de 20 à 40\%, l'activité du précuneus et du cortex cingulaire postérieur (les régions les plus actives à l'éveil et les moins actives sous anesthésie générale ou pendant le sommeil lent profond) est supérieure à celle observée chez des patients en état végétatif [12]. De plus, une meilleure connexion entre le cortex auditif secondaire et les cortex associatifs préfrontaux et temporaux que chez les patients en état végétatif a été observée [3]. Le traitement de l'information se ferait donc à un niveau plus intégré.

Enfin, certaines études ont porté sur le fonctionnement cérébral des patients en syndrome locked-in. Ces patients récupèrent un niveau de conscience normal. Cependant, leur immobilité et les signes de conscience souvent ténus rendent parfois difficile de croire à un retour complet de la conscience. Les observations faites au PET scan montrent toutefois un métabolisme normal et comparable à des sujets sains. Ces résultats confirment donc une bonne récupération de la conscience chez ces patients 'bloqués' dans leur propre corps [12].

\section{Conclusion}

Chacun des états de conscience altérée montre une activité cérébrale spécifique et les observations recueillies grâce à l'utilisation de l'imagerie fonctionnelle ont permis de mieux comprendre ces états pathologiques mais également les processus sous-jacents au phénomène de conscience. Cependant, cette technique ne peut remplacer une évaluation comportementale. Une observation systématique à l'aide d'échelles adaptées reste le moyen principal pour détecter des signes de conscience. Le clinicien doit pouvoir détecter les comportements volontaires et reproductibles, plusieurs évaluations de suite. En outre, la présence de trachéotomie, d'intubation, 
de paralysie ou de lésions (au niveau physique et cérébral) ainsi que de substances curarisantes, anesthésiantes, ou sédatives est susceptible de biaiser l'évaluation et doit être prise en compte. Enfin, le contexte de l'évaluation (en présence de l'équipe soignante ou en présence de la famille), les stimulations utilisées lors de l'évaluation (visuelle/auditive, familière ou non) et les informations rapportées par la famille sont des informations précieuses qui augmentent la qualité de l'évaluation. Ainsi, l'évaluation de la conscience est délicate. Elle est néanmoins plus que jamais indispensable. En effet, comme nous l'avons déjà souligné, le perfectionnement des techniques de réanimation durant ces dernières années permet d'assurer la survie de plus en plus de patients sévèrement cérébrolésés. Certains vont récupérer rapidement mais d'autres seront plus lents et passeront par différents états de conscience altérée avant de récupérer ou non une conscience complète. L'évaluation du fonctionnement cérébral résiduel chez ces patients constitue donc un vrai défi pour les années à venir.

\section{Remerciements}

Cette recherche est soutenue par le Fonds National de la Recherche Scientifique de Belgique (FNRS). SL et SM sont respectivement Chercheur Qualifié et Chargé de Recherches auprès du FNRS.

\section{Références}

[1] American Congress of Rehabilitation Medicine (1995) Recommendations for use of uniform nomenclature pertinent to patients with severe alterations of consciousness. Arch Phys Med Rehabil 76: $205-209$

[2] Ata J, Cook DJ (1998) Prognosis in anoxic and traumatic coma. Crit Care Clin 14: 497-511

[3] Boly M, Faymonville ME, Peigneux P, et al. (in press) Auditory processing in severely brain injured patients: differences between the minimally conscious state and the persistent vegetative state. Archives of Neurology:

[4] Born JD, Hans P, Dexters G, et al. (1982) Practical assessment of brain dysfunction in severe head trauma. Neurochirurgie 28: 1-7

[5] De Volder AG, Goffinet AM, Bol A, et al. (1990) Brain gucose metabolism in postanoxic syndrome. Positron emission tomographic study. Arch Neurol 47: 197-204

[6] Ethics and Humanities Subcommittee of the AAN (1993) Position statement: certain aspects of the care and management of profoundly and irreversibly paralyzed patients with retained consciousness and cognition. Report of the Ethics and Humanities Subcommittee of the American Academy of Neurology. Neurology 43: 222-223

[7] Giacino JT, Ashwal S, Childs N, et al. (2002) The minimally conscious state: Definition and diagnostic criteria. Neurology 58: 349-353.

[8] Giacino JT, Kezmarsky MA, DeLuca J, Cicerone KD (1991) Monitoring rate of recovery to predict outcome in minimally responsive patients. Arch Phys Med Rehabil 72: 897-901

[9] Hattori N, Huang SC, Wu HM, et al. (2003) Correlation of regional metabolic rates of glucose with glasgow coma scale after traumatic brain injury. J Nucl Med 44: 1709-1716

[10] Jennett B (1972) Prognosis after severe head injury. Clin Neurosurg 19: 200-207

[11] Katz RT, Haig AJ, Clark BB, DiPaola RJ (1992) Long-term survival, prognosis, and life-care planning for 29 patients with chronic locked-in syndrome. Arch Phys Med Rehabil 73: 403-408

[12] Laureys S, Berré J, Goldman S (2001) Cerebral function in coma, vegetative state, minimally conscious state, locked-in syndrome and brain death. In: 2001 Yearbook of Intensive Care and Emergency Medicine, Springer-Verlag, Berlin, pp 386-396

[13] Laureys S, Faymonville ME, Degueldre C, et al. (2000) Auditory processing in the vegetative state. Brain 123: 1589-1601.

[14] Laureys S, Faymonville ME, Luxen A, et al. (2000) Restoration of thalamocortical connectivity after recovery from persistent vegetative state. Lancet 355: 1790-1791.

[15] Laureys S, Faymonville ME, Peigneux P, et al. (2002) Cortical processing of noxious somatosensory stimuli in the persistent vegetative state. Neuroimage 17: 732-741.

[16] Laureys S, Lemaire C, Maquet P, Phillips C, Franck G (1999) Cerebral metabolism during vegetative state and after recovery to consciousness. J Neurol Neurosurg Psychiatry 67: 121

[17] Majerus S, Van der Linden M (2000) Wessex Head Injury Matrix and Glasgow/Glasgow-Liège Coma Scale: A validation and comparison study. Neuropsychological Rehabilitation 10: 167-184

[18] Medical Consultants on the Diagnosis of Death (1981) Guidelines for the determination of death. Report of the medical consultants on the diagnosis of death to the President's Commission for the Study of Ethical Problems in Medicine and Biomedical and Behavioral Research,. JAMA 246: 2184-2186

[19] Plum F, Posner JB (1983) The diagnosis of stupor and coma. Davis,F. A., Philadelphia

[20] Rudolf J, Ghaemi M, Haupt WF, Szelies B, Heiss WD (1999) Cerebral glucose metabolism in acute and persistent vegetative state. J Neurosurg Anesthesiol 11: 17-24 
[21] Shiel A, Horn SA, Wilson BA, et al. (2000) The Wessex Head Injury Matrix (WHIM) main scale: a preliminary report on a scale to assess and monitor patient recovery after severe head injury. Clin Rehabil 14: 408-416

[22] Teasdale G, Jennett B (1974) Assessment of coma and impaired consciousness. A practical scale. Lancet 2: 81-84.

[23] The Multi-Society Task Force on PVS (1994) Medical aspects of the persistent vegetative state (1). N Engl J Med 330: 1499-1508

[24] Tommasino C, Grana C, Lucignani G, Torri G, Fazio F (1995) Regional cerebral metabolism of glucose in comatose and vegetative state patients. J Neurosurg Anesthesiol 7: 109-116

[25] Working Party of the Royal College of Physicians (2003) The vegetative state: guidance on diagnosis and management. Clin Med 3: 249-254 
Published in: Réanimation (2004), vol. 13, iss. 5, pp. 368-375 Status: Postprint (Author's version) 